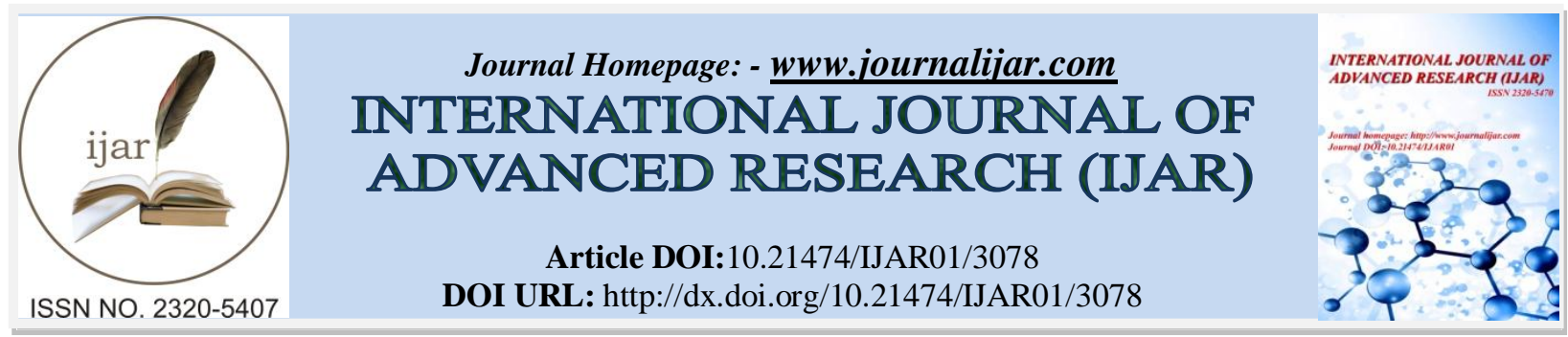

RESEARCH ARTICLE

\title{
A BRIEF OVERVIEW OF MYOFASCIAL TRIGGER POINTS
}

Mansour Abdullah Alshehri, PT B.Sc., M.Sc.

Physiotherapy Department, Faculty of Applied Medical Sciences, Umm Al-Qura University, Saudi Arabia.

\section{Manuscript Info}

\section{Manuscript History}

Received: 06 November 2016

Final Accepted: 31 December 2016

Published: January 2017

Copy Right, IJAR, 2016,. All rights reserved.

\section{Introduction:-}

It has usually been believed that myofascial trigger points (MTrPs) are significant pathologies in the muscles: sometimes these points become painful and extremely irritating. MTrPs are commonly described as the presence of local tenderness due to the existence of a contraction knot in a taut band of the muscle tissue (Figure 1). These points are painful and may produce referred pain, either spontaneously or due to direct pressure. MTrPs can be regarded as one of the most frequent causes of pain and disability in the muscular and skeletal system (Cummings and Baldry, 2007). It is desirable to clarify theories and explanations regarding the MTrP mechanism and its relation to pain?

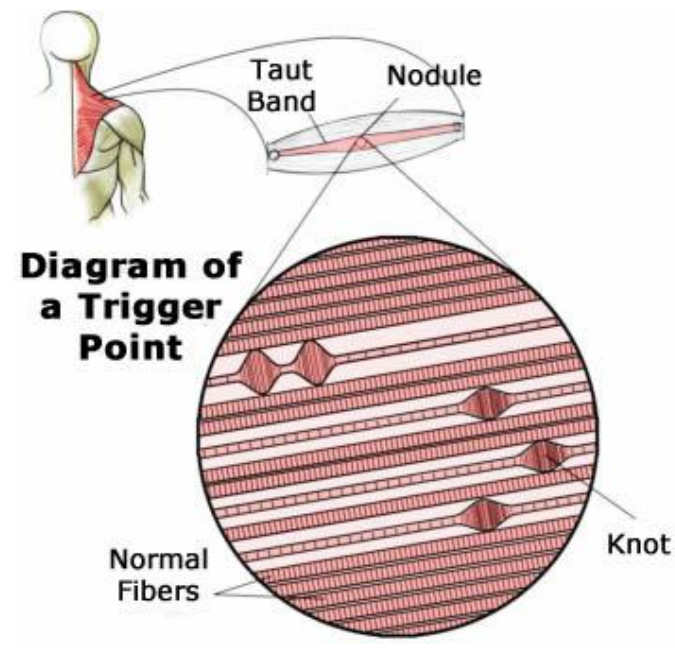

Fig 1:-Diagram of a Myofascial Trigger Point (taken from http://www.healbodypain.com)

It seems possible that MTrPs are not a serious condition; however, it is important to clearly understand the concept of the MTrP pain mechanism. MTrPs can be divided into two main types: the first is referred to as a 'latent trigger point', while the second is called an 'active trigger point'. Latent trigger points are inactive points without spontaneous pain, but which might be painful as a result of deep and direct compression. On the other hand, active trigger points are painful and spontaneous, and may associated with referred pain. These points might also present

Corresponding Author:-Mansour Abdullah Alshehri.

Address:-Physiotherapy Department, Faculty of Applied Medical Sciences, Umm Al-Qura University, 
some other symptoms such as muscle weakness, a limited range of motion in the affected area and temperature alterations (Huguenin, 2004).

It is possible for a latent trigger point to become an active point. Many factors, such as traumatic injuries, muscle overuse, poor posture, psychological stress and systemic pathology may contribute to the development of a local palpable latent MTrP. Therefore, a latent MTrP could became an active MTrP if subjected to mechanical stress or to other hurtful factors (Saxena et al., 2015). An active MTrP may spontaneously recover, return to the latent stage, or continue without progression. Moreover, an active MTrP could remain in a dynamic state, so it can change from a latent MTrP into an active MTrP and back again in a vicious cycle, this process is probably controlled by stressors (Figure 2). MTrPs can be also classified as primary and secondary MTrPs. A primary MTrP occurs due to the overloading or overuse of specific muscle tissue, where its activation not caused by the action of another muscle. Secondary (satellite) MTrPs develop due to mechanical stress and/or neurogenic inflammation as a result of the activity of a primary trigger point. The activity of nociceptive receptors in various structures (somatic or visceral) can develop secondary MTrPs (Simons et al., 1999).

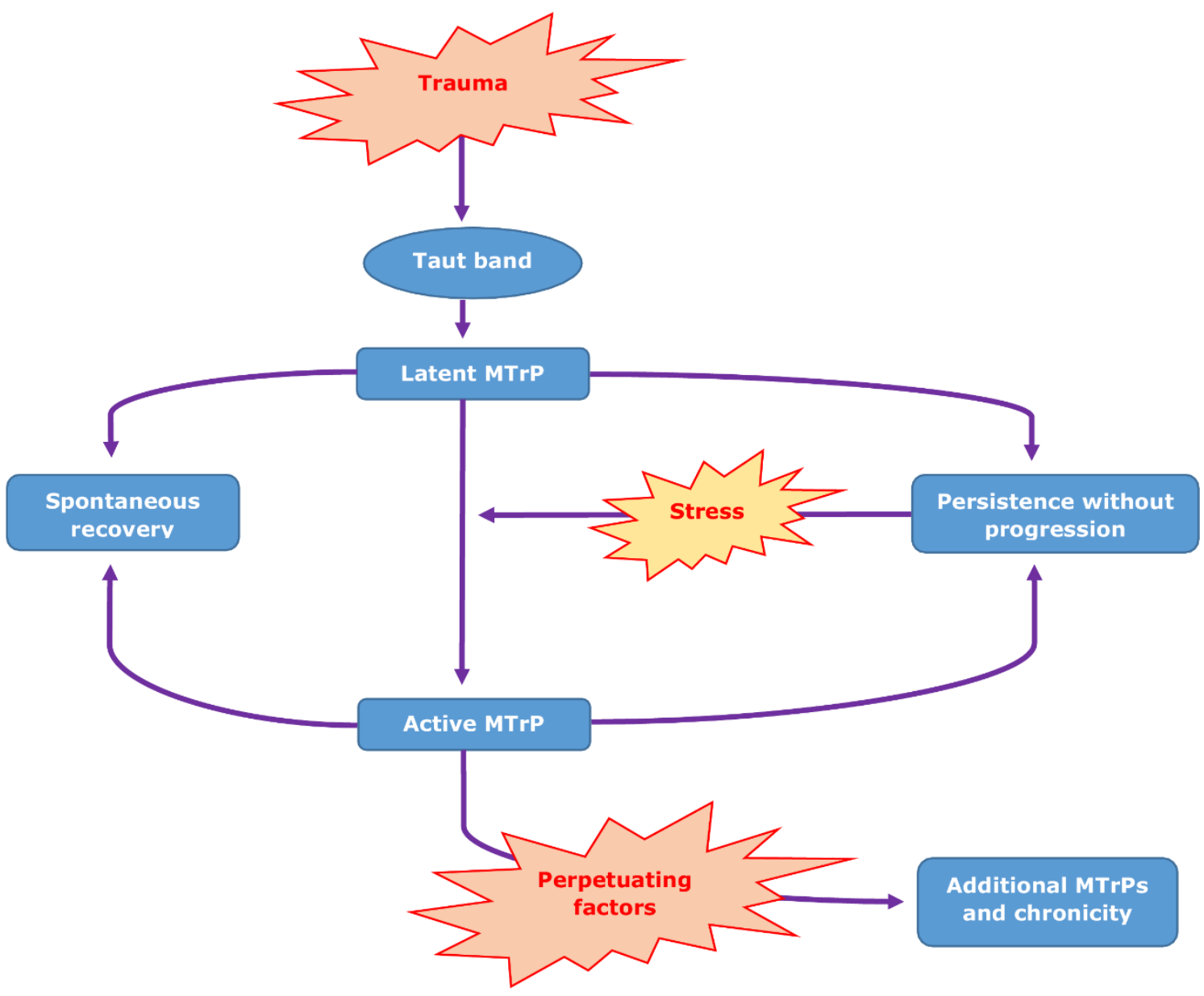

Fig 2:-Vicious cycle of myofascial trigger points (adapted from Saxena et al., 2015)

\section{Pathophysiology:-}

There is a heated debate regarding the pathophysiology of MTrPs, specifically, a number of hypotheses exist concerning its mechanism. For example, an integrated hypothesis suggests that MTrPs are caused by overloading or overuse of a muscle that might cause micro-trauma, this might lead to a release of acetylcholine (ACh). An increased amount of $\mathrm{ACh}$ contributes to a contraction knot, which is an area where there is a localized shortening in 
the sarcomere. This contraction is sustained, and leads to local ischaemia and hypoxia (Simons, 2004). Consequently, a loss of the energy supply (oxygen) leads to a release of sensitizing noxious substances, which may be the cause of the pain associated with MTrPs. This hypothesis is called the 'energy crisis theory', and has been widely accepted by many healthcare professionals. Another, similar hypothesis exists, stating that blood vessel compression resulting from a continued contraction knot causes local hypoperfusion (Gerber, 2011). As a result, the ischemic tissue is usually associated with pain and tenderness.

In contrast, to the above theories, other theories exist that focus more on neurological models. These theories propose that neuro tissue or process involvement are the major causes of myofascial pain, whereas the trigger point is a relatively minor phenomenon. One of their explanations is that the properties of myofascial pain based on myofascial trigger points are not discernible from those of neural pain. Therefore, these theories state that the main cause of MTrPs symptoms is due to disorders of the nervous system such as neuritis (inflammation of the nerve), allodynia (pain sensations from a non-painful stimulus) or hyperalgesia (exaggerated sensitivity to pain) (Quintner, 2014).

Numerous studies have discussed the factors that cause or maintain myofascial pain. Saxena et al. (2015) identified three categories of perpetuating factors that may have a negative effect on the body; these could lead to the development and increase in the number of MTrPs, which might eventually also develop myofascial pain syndrome. These factors are divided into three main components: structural factors (such as scoliosis, functional leg length inequality and osteoarthrosis of the main joints); ergonomic factors (for example, poor posture and work-related activities); and medical factors (such as hormonal imbalance, nutritional deficiencies and infectious diseases). However, these categories do not take into account the psychosocial factors that probably play an important role in developing MTrPs. It has been acknowledged that psychosocial factors such as anxiety and depression are frequently found in patients with chronic pain (Kuch, 2001). Therefore, muscle tension might cause fatigue, and with high loads in the muscle tissue, this could lead to the development of MTrPs. Therefore, it is necessary consider all possible factors in depth.

\section{Diagnosis:-}

The examination of MTrPs has not been supported by high quality evidence. MTrPs are assumed to be localized, although they are identified as a result clinical examination by clinicians, these examination methods do not follow sufficiently rigorous criteria, and other therapists undertaking such an examination might identify different MTrPs or none at all. Another issue is that these points can be palpated only if located in the superficial layer of the muscle tissue, or where they are associated with areas of localized muscle shortening. Thus, it remains questionable whether therapists can differentiate between muscle stiffness, localized adhesions, allodynia, and tender points without a contraction knot from MTrPs. Therefore, a high level of manual skill is required to detect these points accurately. There are four major clinical features of MTrPs as follows (Cummings and Baldry, 2007):

- A tender point in the taut muscle tissue

- A possible pattern of pain distribution

- Patient/client recognition of pain dueing continued pressure over MTrPs

- A local twitch response (LTR), which is a temporary and a quick contraction of the muscle fibre during pincer palpation of MTrPs. LTR could be visible or palpable, or both.

MTrPs can be palpated and assessed in three ways: direct pressure, flat palpation and pincer palpation. The first two methods are used for superficial muscle tissue, while the third is used for deep muscle tissue (Simons et al., 1999). However, the force and accuracy of performance that is applied during the assessment may influence the process and production of referred pain; as a result, this may affect the judgment of the clinician regarding the diagnosis. Complementary and instrumental testing methods such as ultrasound, electromyography, algometry and thermography are still controversial, and their ability to diagnose MPS and detect MTrPs have not been confirmed, due to their lack of sensitivity and specificity (Vázquez-Delgado et al., 2009). In practical, these methods are usually employed simply to confirm the clinician's diagnosis.

As a result, the relationship between MTrPs and myofascial pain syndrome (MPS) is a controversial topic, for many reasons. There are no definite, validated and significant diagnostic criteria for detecting trigger points in the body. Moreover, the overlapping of MPS symptoms with those of other musculoskeletal pain disorders may lead to confusion in the diagnosis. Furthermore, most of the evidence, which supports the idea that MPS is based on trigger points, relates to low-quality studies or ones conducted using only small groups, which may not allow us to 
generalize the results. Nevertheless, some recent studies have investigated other causes of MPS, such as central sensitization (Giamberardino et al., 2011; Quintner, 2014).

\section{Conclusion:-}

MTrPs consist of two types, active or latent, and changes between these states may form a vicious cycle, which is usually controlled by stressors. Many theories exist regarding the causes of MTrPs and how they produce pain, but no theory has been significantly confirmed and stand out above the other theories. Several perpetuating factors might play a primary or secondary role in the development of MTrPs or might activate latent MTrPs. Clinicians should consider these issues when using any interventions that are based on the detection of MTrPs. Future studies need to investigate the relationship between pain and MTrPs, such research should involve patients with a variety of musculoskeletal disorders, in order to provide an adequate recommendation.

\section{References:-}

1. Cummings, M. and Baldry, P. (2007): Regional myofascial pain: diagnosis and management. Best Pract. Res. Clin. Rheumatol., 21(2): 367-387.

2. Gerber, N.L., Sikdar, S., Hammond, J. and Shah, J. (2011): A Brief Overview and Update of Myofascial Pain Syndrome and Myofascial Trigger Points. Journal of Spinal Research Foundation, 6(1): 55-64.

3. Giamberardino, M.A., Affaitati, G., Fabrizio, A. and Costantini, R. (2011): Myofascial pain syndromes and their evaluation. Best Pract. Res. Clin. Rheumatol., 25(2): 185-198.

4. Huguenin, L.K. (2004): Myofascial trigger points: the current evidence. Phys. Ther. Sport., 5(1): 2-12.

5. Kuch, K. (2001): Psychological factors and the development of chronic pain. Clin. J. Pain., 17(4): 33-38.

6. Quintner, J. L., Bove, G. M., \& Cohen, M. L. (2014). A critical evaluation of the trigger point phenomenon. Rheumatology, 54(3): 392-399.

7. Saxena, A., Chansoria, M., Tomar, G. and Kumar, A. (2015): Myofascial pain syndrome: an overview J. Pain. Palliat. Care. Pharmacother., 29(1): 16-21.

8. Simons, D.G. (2004): Review of enigmatic MTrPs as a common cause of enigmatic musculoskeletal pain and dysfunction. J. Electromyogr. Kinesiol., 14, 95-107.

9. Simons, D.G., Travell, J.G. and Simons, L.S. (1999): Travell\& Simons' myofascial pain and dysfunction the trigger point manual. Second edition, Williams \& Wilkins, Baltimore, USA.

10. Vázquez-Delgado, E., Cascos-Romero, J. and Gay-Escoda, C. (2009): Myofascial pain syndrome associated with trigger points: a literature review. (I): Epidemiology, clinical treatment and etiopathogeny. Medicina Oral, Patología Oral y CirugíaBucal 14(10): 494-498. 